\title{
Site-specific Phenotype of Atherosclerotic Lesions According to Their Location Within the Coronary Tree - a CCTA-based Study of Vulnerable Plaques
}

\author{
Diana Opincariu ${ }^{1}$, Nora Rat ${ }^{1,2}$, Andras Mester ${ }^{1}$, Roxana Hodas ${ }^{1}$, Daniel Cernica ${ }^{2}$, Dan Pasaroiu ${ }^{2}$, \\ Mihaela Ratiu' ${ }^{3}$ Monica Chitu ${ }^{2,3}$, Istvan Kovacs ${ }^{2,3}$, Imre Benedek ${ }^{2,3}$, Theodora Benedek ${ }^{1,2}$ \\ 1 "George Emil Palade" University of Medicine, Pharmacy, Science and Technology, Târgu Mureș, Romania \\ ${ }^{2}$ Clinic of Cardiology, Emergency Clinical County Hospital, Târgu Mureș, Romania \\ ${ }^{3}$ Center of Advanced Research in Multimodal Cardiac Imaging, Cardio Med, Târgu Mureș, Romania
}

\section{ABSTRACT}

Background: The evaluation of site-specific phenotype according to the topographic location of atherosclerotic lesions within the coronary tree has not been studied so far. The present study is based on the premise that the location of coronary plaques can influence their composition and degree of vulnerability. Aim: To evaluate different phenotypes of vulnerable coronary plaques across the three major coronary arteries in terms of composition, morphology, and degree of vulnerability, in patients with chest pain and low-to-intermediate probability of coronary artery disease, using coronary computed tomography angiography (CCTA) and a complex plaque analysis. Material and methods: This was a cross-sectional study on 75 subjects undergoing CCTA for chest pain, who presented at least one vulnerable coronary plaque (VP), defined as the presence of $\geq 1$ CT vulnerability marker (low attenuation plaque, napkin-ring sign, spotty calcifications, positive remodeling). The study included per plaque analysis of 90 vulnerable coronary lesions identified in various locations within the coronary tree as follows: $n=30$ VPs in the left anterior descending artery (LAD), $n=30$ VPs in the circumflex artery (CXA), and $n=30$ VPs in the right coronary artery (RCA). Results: The RCA exhibited significantly longer VPs $(p=0.001)$, with the largest volume $(p=0.0007)$ compared to those arising from the LAD and CXA. Vulnerable plaques located in the LAD exhibited a significantly more calcified phenotype (calcified volume: LAD $-44.07 \pm 63.90 \mathrm{~mm}^{3} \mathrm{vs}$. CXA $12.40 \pm 19.65 \mathrm{~mm}^{3}$ vs. RCA $\left.-33.69 \pm 34.38 \mathrm{~mm}^{3}, \mathrm{p}=0.002\right)$. Plaques from the RCA presented a more non-calcified phenotype, with the largest non-calcified $(\mathrm{p}=0.002)$, lipid rich $(\mathrm{p}=$ $0.0005)$, and fibrotic volumes $(p=0.003)$. Low-attenuation plaques were most frequent in the RCA ( $\mathrm{p}=0.0009)$, while the highest vulnerability degree was present in lesions located in the LAD, which presented the highest number of vulnerability markers per plaque $(p=0.01)$. Conclusions: Vulnerable plaques arising from the right coronary artery are longer, more voluminous and with larger lipid and non-calcified content, whereas those located in the left anterior descending artery present a higher volume of calcium, but also a higher degree of vulnerability. The least vulnerable lesions were present in the circumflex artery.

Keywords: coronary artery disease, coronary CT angiography, vulnerability degree, plaque location

\section{ARTICLE HISTORY}

Received: April 30, 2021

Accepted: May 15, 2021

\section{CORRESPONDENCE}

Daniel Cernica

Str. Gheorghe Marinescu nr. 50 540139 Târgu Mureș, Romania

Tel: +40372653100

E-mail: daniel.cernica@gmail.com 
Dan Pasaroiu • Str. Gheorghe Marinescu nr. 38, 540136 Târgu Mureș, Romania. Tel: +40 265215 551, E-mail: dan.pasaroiu@yahoo.com Mihaela Ratiu •Str. 22 Decembrie 1989 nr. 76, 540124 Târgu Mureș, Romania. Tel: +40 265 217 333, E-mail: d_a_mihaela@yahoo.com Monica Chitu •Str. 22 Decembrie 1989 nr. 76, 540124 Târgu Mureș, Romania. Tel: +40 265217 333, E-mail: iulia.chitu@yahoo.com Istvan Kovacs · Str. 22 Decembrie 1989 nr. 76, 540124 Târgu Mureș, Romania. Tel: +40 265 217 333, E-mail: kov_istvan@yahoo.com Imre Benedek· Str. Gheorghe Marinescu nr. 38, 540136 Târgu Mureș, Romania. Tel: +40 265215 551, E-mail: imrebenedek@yahoo.com Theodora Benedek • Str. Gheorghe Marinescu nr. 38, 540136 Târgu Mureș, Romania. Tel: +40 265 215 551, E-mail: theodora.benedek@gmail.com

\section{BACKGROUND}

Coronary artery disease (CAD), one of the main causes of mortality in the developed world, is caused by atherosclerotic plaque build-up over the course of several years, under the influence of various local and systemic factors. ${ }^{1}$ Being a chronic disease, CAD is initiated by inflammation within the vessel wall, followed by lipid deposition and the development of complex plaques, intracoronary thrombosis, and acute coronary syndromes (ACS), which are associated with increased morbidity and mortality. ${ }^{2}$ There are three postulated mechanisms than can lead to ACS: plaque rupture, erosion, and calcified nodules. All three pathophysiological scenarios are associated with intracoronary thrombus formation and myocardial ischemia. ${ }^{3-5}$

The concept of vulnerable plaque (VP) was developed in an attempt to identify atherosclerotic lesions with a high propensity to rupture. The current definition of VP includes lesions that are prone to becoming culprit, thus leading to ACS and death. ${ }^{6,7}$ A VP is characterized by a large lipid-filled necrotic core, a thin fibrous cap $(<65$ $\mu \mathrm{m})$, eccentric vascular remodeling, neovascularization and intraplaque hemorrhage, as well as microcalcifications within the necrotic core. ${ }^{6,8,9}$

Several invasive imaging techniques, such as intravascular ultrasound (IVUS) and optical coherence tomography (OCT), have been established as reference diagnostic methods for the visualization of coronary VPs. Their disadvantages arise from their invasive nature, higher costs, and increased complication rates. ${ }^{10,11}$ However, coronary computed tomography angiography (CCTA) has emerged as the method of choice for imaging atherosclerosis, being cost-effective, noninvasive, widely available, with an increased spatial resolution, and high specificity and sensitivity. According to the latest guideline of the European Society of Cardiology for the management of stable CAD, CCTA is the paraclinical method of choice for evaluating vessel anatomy and severity of atherosclerotic lesions in subjects with low-to-intermediate pre-test probability for CAD. ${ }^{12}$

CCTA differentiation of plaque components, based on the measurement of Hounsfield units across different plaque regions, has allowed comprehensive characteriza- tion and identification of VPs. A high-risk plaque is characterized by a thin-cap fibroatheroma, a large necrotic core (the CCTA correspondent of low-attenuation regions within the plaque), heterogeneous calcifications (identified on CCTA as spotty calcium), and outward vascular remodeling. ${ }^{13-15}$ On the other hand, the landmark of plaque stabilization is a thick-cap fibroatheroma, dense calcium deposits, and inward vascular remodeling. ${ }^{16,17}$ There are four known CCTA vulnerability markers that have been validated with invasive imaging studies as well as autopsy studies, which include: low-attenuation plaque (LAP), positive remodeling (PR), spotty calcifications (SC), and the napkin-ring sign (NRS) ${ }^{18-20}$

There is scarce data regarding plaque characteristics according to their topographic location within the coronary tree. However, previous epidemiological data have shown that atherosclerotic lesions are not uniformly distributed between the right and left coronary artery. ${ }^{21-24}$ First, it has been proven that initial atherosclerotic lesions are more frequent in the left compared to right coronary artery, ${ }^{22}$ while the circumflex artery is less likely to be affected by plaque build-up. ${ }^{25}$ Second, Despite similar exposure to systemic cardiovascular risk factors, there are some vascular regions that are more prone to present atherosclerotic lesions, including proximal areas, bifurcations, and curvatures. This suggests that local factors, such as hemorheological and hemodynamical characteristics, influence plaque development and progression. ${ }^{23,24}$ Last, but not least, it appears that plaque topography may impact patient outcomes and the risk of future cardiovascular events. Plaques situated in the left coronary artery and left coronary dominance have been associated with higher risk of adverse events. This was not true however, for plaques located in the right coronary arterial system. ${ }^{25,27}$

The evaluation of site-specific phenotype according to the topography of atherosclerotic lesions within the coronary tree has not been studied so far. This, in relation to the importance of early characterization and treatment of vulnerable plaques, can provide great benefits in preventing the occurrence of ACS. Several characteristics related to vessel geometry, coronary shear stress, but also local inflammation can contribute to the process of plaque destabilization. The present study is based on the premise 
that the location of atherosclerotic plaques in the three main coronary arteries can influence their composition and degree of vulnerability.

\section{AIM}

We sought to evaluate different phenotypes of vulnerable coronary plaques across the three major coronary arteries in terms of composition, morphology, and degree of vulnerability in patients with chest pain and low-to-intermediate probability of $\mathrm{CAD}$, with the use of CCTA and a complex plaque analysis.

\section{MATERIALS AND METHODS}

\section{STUDY DESIGN AND POPULATION}

This was a cross-sectional observational study that included 75 patients presenting with chest pain, with lowto-intermediate clinical likelihood for CAD, who underwent 128-slice CCTA for assessment of coronary anatomy, atherosclerosis, and plaque analysis. All patients presented at least one VP in the coronary tree, defined as the presence of one or more of the following CCTA vulnerability markers: LAP, PR, SCs, and NRS.

The exclusion criteria consisted in the presence of high degree of coronary calcifications, irregular heart rate or inability to achieve a heart rate $<65 \mathrm{bpm}$, morbidly obese patients, failure to obey breath-hold commands, or any other conditions which may interfere with proper image acquisition or quality. Other exclusion criteria comprised: detection of other causes of chest pain than CAD; a history of myocardial infarction; the presence of coronary stents or other intracardiac devices; a high clinical likelihood for $\mathrm{CAD}$, severe symptoms, or acute coronary syndromes that required invasive coronary angiography.

All the study procedures were performed according to the good clinical practice guidelines and the Declaration of Helsinki, and were approved by the Ethics Committee of the institution where the study was conducted. All study participants consented to the study prior to enrollment.

\section{CT ACQUISITION PROTOCOL AND IMAGE POST-PROCESSING}

For image acquisition, all subjects underwent coronary CCTA with the use of a 128-slice Somatom Definition scanner (Siemens Healthcare, Germany). The scanning was performed by using retrospective gating at a heart rate of $<65 \mathrm{bpm}$, with $120 \mathrm{kV}$ tube voltage, gantry ro- tation time of 0.33 seconds, $128 \times 0.6$ collimation. For patients with a resting heart rate of over $65 \mathrm{bpm}$, intravenous or oral beta blocker was administered. Initially, the acquisition includes a native scanning for evaluation of coronary calcium, followed by the administration of 80-100 $\mathrm{mL}$ of iodine-based contrast solution according to body weight, with a $50 \mathrm{~mL}$ saline chase at a flow rate of $5.5 \mathrm{~mL} / \mathrm{s}$, during inspiratory breath-hold. All the acquired scans were stored in a dedicated imaging database for offline post-processing. Figure 1 illustrates a CCTA image of a vulnerable plaque located in the right coronary artery (RCA), exhibiting several vulnerability markers, with a significant degree of stenosis (A) and
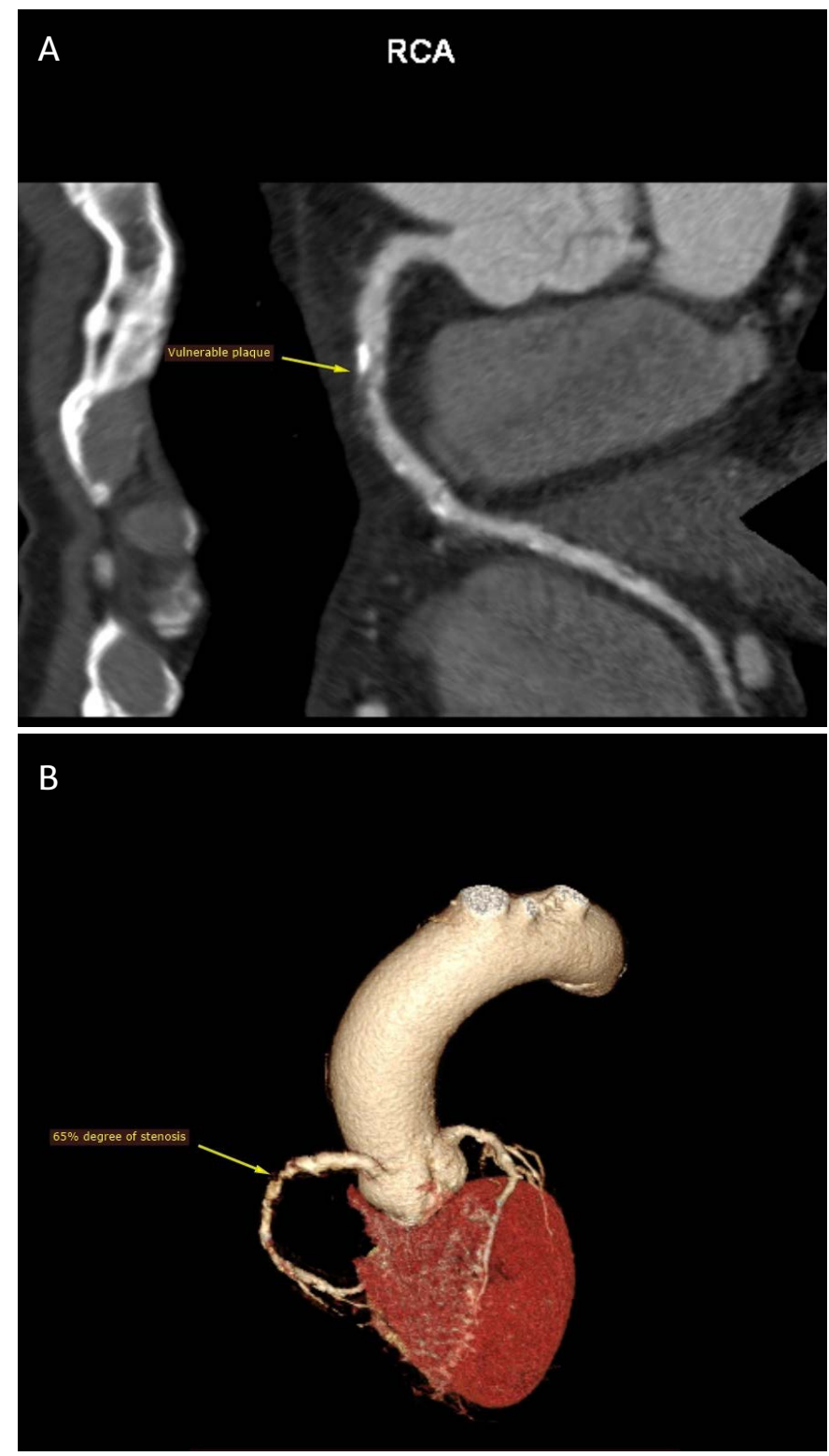

FIGURE 1. CCTA image of a vulnerable plaque located in the RCA (A) and 3D reconstruction showing a $65 \%$ degree of stenosis (B) 
TABLE 1. Comparison of parameters indicating vulnerable plaque morphology across the three main branches of the coronary tree

\begin{tabular}{lcccc}
\hline & LAD & CXA & RCA & p value \\
\hline Plaque length (mm) & $18.67 \pm 5.49$ & $15.48 \pm 3.73$ & $20.47 \pm 5.97$ & 0.001 \\
Degree of stenosis (\%) & $57.77 \pm 8.62$ & $54.50 \pm 11.25$ & $59.63 \pm 10.42$ & 0.02 \\
MLA $\left(\mathrm{mm}^{2}\right)$ & $0.03 \pm 0.01$ & $0.05 \pm 0.02$ & $0.03 \pm 0.01$ & 0.01 \\
Eccentricity index & $0.37 \pm 0.19$ & $0.34 \pm 0.26$ & $0.39 \pm 0.22$ & 0.4 \\
Remodeling index & $1.05 \pm 0.30$ & $1.12 \pm 0.15$ & $1.05 \pm 0.23$ & 0.1 \\
Plaque volume $\left(\mathrm{mm}^{3}\right)$ & $187.9 \pm 86.03$ & $146.9 \pm 102.4$ & $248.1 \pm 110.4$ & 0.0007 \\
\hline
\end{tabular}

the three-dimensional reconstruction showing a significant degree of stenosis (B).

All the acquired images were post-processed on a remote offline dedicated computer with the use of the Syngo Via Frontier (Siemens Healthineers, Erlangen Germany) software system for VP analysis. This comprised the evaluation of plaque location, morphology (length, volume, degree of stenosis), composition (lipid rich plaque - with a CT density of -100 to $-30 \mathrm{HU}$; fibrotic plaque -30 to $40 \mathrm{HU}$; calcified plaque -400 to $-950 \mathrm{HU}$ ), as well as the vulnerability degree based on the presence of the aforementioned CT markers (LAP - CT density of $<-30 \mathrm{HU}, \mathrm{PR}$ - remodeling index of $>1.1$, SCs, NRS).

\section{STATISTICAL ANALYSIS}

After coronary plaque analysis, all the exhibited parameters were stored in a dedicated electronic database and were statistically analyzed using the GraphPad Prism 6 software. The study included a per plaque analysis of 90 vulnerable coronary lesions identified in various locations within the coronary tree as follows: $\mathrm{n}=30$ VPs in the left anterior descending artery (LAD), $\mathrm{n}=30$ VPs in the circumflex artery (CXA), and $n=30$ VPs in the RCA. The one-way ANOVA test was used for comparison of quantitative variables and the Chi square test for binary data, and the statistical significance of the study was set at an alpha of 0.05 .

\section{RESULTS}

\section{PLAQUE MORPHOLOGY}

Coronary plaque analysis revealed that the RCA exhibited significantly longer VPs (LAD $-18.67 \pm 5.49 \mathrm{~mm}$ vs. CXA $-15.48 \pm 3.73 \mathrm{~mm}$ vs. RCA $-20.47 \pm 5.97 \mathrm{~mm}, \mathrm{p}=$ 0.001 ), with larger volumes (LAD $-187.9 \pm 86.03 \mathrm{~mm}^{3} \mathrm{vs}$. CXA $-146.9 \pm 102.4 \mathrm{~mm}^{3}$ vs. RCA $-248.1 \pm 11.4 \mathrm{~mm}^{3}, \mathrm{p}=$ 0.0007) when compared to plaques located in the LAD and CXA. The remodeling $(p=0.1)$ and eccentricity indices $(p=$
0.4) were not significantly different between VPs located across the three main coronary vessels (Table 1 ). The largest degree of stenosis was present for VPs located in the RCA (LAD - $57.77 \pm 8.62 \mathrm{~mm}$ vs. CXA $-54.50 \pm 11.25 \mathrm{~mm}$ vs. RCA $-59.63 \pm 10.42 \mathrm{~mm}, \mathrm{p}=0.02$ ) (Table 1 ).

\section{PLAQUE COMPOSITION}

Vulnerable plaques located in the LAD exhibited a significantly more calcified phenotype (calcified volume: LAD $44.07 \pm 63.90 \mathrm{~mm}^{3}$ vs. CXA $-12.40 \pm 19.65 \mathrm{~mm}^{3}$ vs. RCA $-33.69 \pm 34.38 \mathrm{~mm}^{3}, \mathrm{p}=0.002$ ). Conversely, VPs identified in the RCA presented a more non-calcified phenotype, illustrated by quantification of non-calcified (LAD - 143.8 $\pm 76.02 \mathrm{~mm}^{3}$ vs. CXA $-134.5 \pm 102.2 \mathrm{~mm}^{3}$ vs. RCA -214.4 $\left.\pm 99.67 \mathrm{~mm}^{3}, \mathrm{p}=0.002\right)$, lipid rich (LAD $-14.95 \pm 22.69$ $\mathrm{mm}^{3}$ vs. CXA $-6.44 \pm 13.42 \mathrm{~mm}^{3}$ vs. RCA $-16.07 \pm 15.74$ $\left.\mathrm{mm}^{3}, \mathrm{p}=0.0005\right)$, and fibrotic volumes (LAD $-128.9 \pm$ $66.10 \mathrm{~mm}^{3}$ vs. CXA $-128.1 \pm 91.56 \mathrm{~mm}^{3}$ vs. RCA $-198.3 \pm$ $92.34 \mathrm{~mm}^{3}, \mathrm{p}=0.003$ ), as shown in Figure 2.

\section{VULNERABILITY DEGREE}

When comparing the CT vulnerability markers between lesions located in the three arteries, there were no significant differences between plaques located in the LAD, CXA, and RCA in regards to the presence of the napkin-ring sign $(\mathrm{p}=0.4)$, spotty calcifications $(\mathrm{p}=0.6)$, or positive remodeling $(\mathrm{p}=0.8)$. However, low-attenuation plaques were significantly more frequent in lesions located in the RCA $(p=0.0009)$. The highest vulnerability degree was present in lesions located in the $\mathrm{LAD}$, which presented the highest number of vulnerability markers per plaque $(\mathrm{p}=$ o.01) (Table 2).

\section{DISCUSSIONS}

The present research aimed to analyze the site-specific phenotype of vulnerable plaques according to their loca- 
A

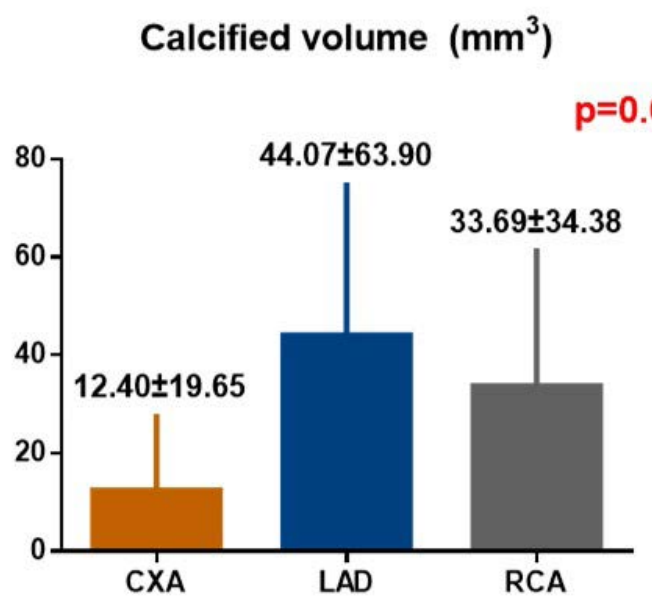

C

Lipid rich volume $\left(\mathrm{mm}^{3}\right)$

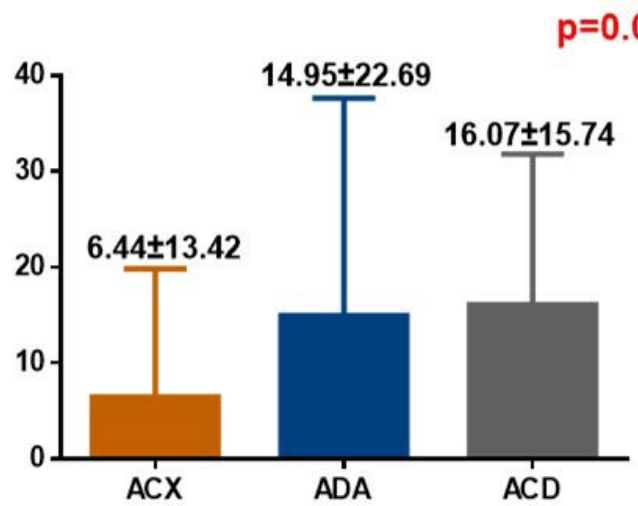

B

Non-calcified volume $\left(\mathrm{mm}^{3}\right)$

$p=0.001$

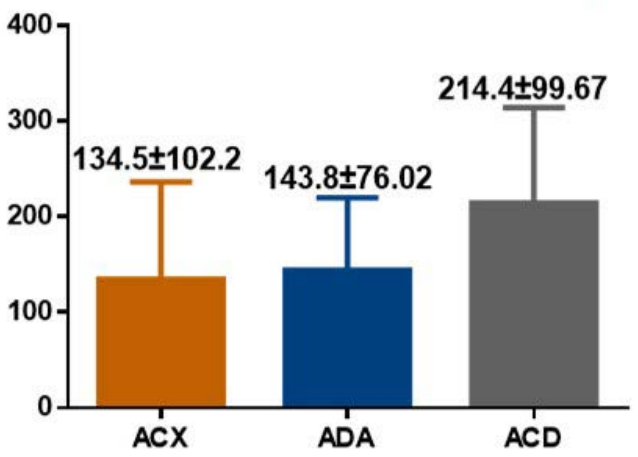

D

Fibrotic volume $\left(\mathrm{mm}^{3}\right)$

$p=0.003$

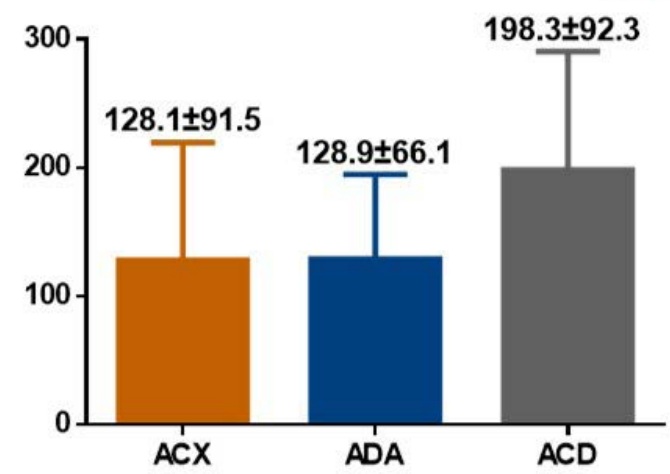

FIGURE 2. Analysis of plaque composition for calcified volume (A), non-calcified volume (B), lipid-rich volume (C), fibrotic volume (D) comparative analysis between vulnerable plaques located in the LAD, CXA, and RCA

tion within the coronary tree, by using CCTA imaging. CCTA provides detailed information on coronary tree anatomy, degree of stenosis and severity, but also morphology and composition of coronary atherosclerotic plaques. There is little data regarding the difference between the three main coronary arteries, and most available studies focus on hemorheological and hemodynamic aspects, but not on the morphological characteristics of lesions.
Previous epidemiological data have shown that atherosclerotic lesions are not uniformly distributed between the right and left coronary arteries. Single vessel disease has been found more frequently in the left compared to the right coronary artery (35\% versus $6.5 \%) .{ }^{22}$ In addition, an IVUS study on transplanted hearts (mean age $33.4 \pm 13.2$ years) found that the most frequent location of atherosclerotic lesions was in the LAD, followed by the RCA and the CXA. ${ }^{28}$

TABLE 2. Analysis of vulnerability degree across the three main coronary arteries

\begin{tabular}{lcccc}
\hline & LAD & CXA & RCA & p value \\
\hline NRS, n (\%) & $5(16.6 \%)$ & $2(6.66 \%)$ & $3(10 \%)$ & 0.4 \\
SC, n (\%) & $18(60 \%)$ & $18(60 \%)$ & $16(53.3 \%)$ & 0.6 \\
PR, n (\%) & $14(46.6 \%)$ & $17(56.6 \%)$ & $14(46.6 \%)$ & 0.8 \\
LAP, n (\%) & $18(60 \%)$ & $8(26.6 \%)$ & $22(73.3 \%)$ & 0.0009 \\
Number of vulnerability markers & $2.2 \pm 0.8$ & $1.6 \pm 0.7$ & $1.8 \pm 0.6$ & 0.01 \\
\hline
\end{tabular}


Therefore, our study hypothesized that plaque composition and degree of vulnerability is influenced by the plaques' location across the three main coronary branches. For this we conducted a comparative per plaque analysis of lesions located in the LAD, CXA, and RCA that had presented at least one of the four CCTA vulnerability markers (LAP, NRS, SCs, PR). The main results indicated that VPs located in the RCA were soft, more voluminous, and with a higher lipid-rich and non-calcified content. On the other hand, those situated in the LAD were more calcified, but also more vulnerable, as expressed by a higher number of vulnerability markers per lesion.

Even if exposure to systemic risk factors is similar, some arterial regions are more prone to plaque build-up. Several topographical characteristics, including branching sites, curvatures, and proximal regions, are more likely to present atheroma, thus pinpointing the role of local factors that contribute to plaque development. Local factors that may influence the evolution of atherosclerosis include the endothelial shear stress, the molecular flow viscosity and wall stress, in association with vessel geometry, curvature, and tortuosity. ${ }^{23,24}$ A proof of concept study published in 2015, based on computational fluid dynamics, found that the LAD is characterized by higher shear stress values compared to the CXA and RCA, for both atherosclerotic and non-atherosclerotic segments. The molecular viscosity and curvature were the highest in segments analyzed from the CXA. ${ }^{21}$ In our study, the analysis of plaque morphology across the three coronary branches revealed that VPs with the higher length, volume, and degree of stenosis were located in the RCA, followed by the LAD and the CXA.

Vascular geometry in association with hemodynamic characteristics of coronary blood flow, systemic risk factors, and local inflammation, all modulate the atherogenic process in predisposed topographical arterial sites. This also leads to alteration of plaque composition and vulnerability. Low shear stress promotes plaque development and growth, while high coronary shear stress leads to plaque destabilization. However, the pulsatile flow and oscillations in hemorheology result in the onset of atherosclerosis. ${ }^{21}$ This could be the explanation as to why in our study, the highest calcified volume was present in the LAD, but simultaneously these were also the most vulnerable. Although increased calcification has been proven as a sign of plaque stability, it may also indicate increased vulnerability, in accordance to calcium distribution within the coronary lesion. This is shown in our study by the presence of spotty calcifications, which were most expressed in lesions located in the LAD.
The presence of any of the CCTA vulnerability markers (LAP, PR, NRS, SCs) in coronary plaques is associated with increased risk of major adverse cardiovascular events, and a higher number of vulnerability markers per lesion exponentially increases this risk..$^{30,31}$ In our study, the NRS and SCs were most frequent in LAD lesions, although the difference did not reach statistical significance. Conversely, low-attenuation plaques were more frequently found in the RCA, which was also confirmed by a higher lipidrich and non-calcified content. The least vulnerable lesions, with the lowest number of vulnerability markers per plaque, but with the highest expression of positive remodeling were located in the CXA.

\section{FUTURE DIRECTIONS}

Several future research directions can arise from the current proof of concept study. Our results show a clear difference in plaque morphology according to location within the coronary tree. However, one of the future directions is to conduct a similar analysis which includes also the hemorheological and hemodynamical characteristics of coronary flow, the proximal versus distal location, and also the effect of coronary dominance. In addition, the role of plaque location on patient outcomes has yet to be established.

Plaque topography may have prognostic value in patients with CAD. A CCTA study on 1,425 patients has shown that stenoses located in the left coronary arterial system were associated with an increased risk of events, but this was not true for lesions within the right coronary artery. Their results also stated that left dominance associated a higher risk of mortality over the two-year follow-up period. ${ }^{27}$ Another CCTA-based study derived from the CONFIRM Cohort found a higher incidence of CAD, obstructive and non-obstructive, in patients with left compared to right coronary dominance, although the presence of multivessel disease did not differ among groups. ${ }^{26}$ Additionally, the prognosis of CAD patients is directly associated by the degree of myocardial ischemia in the subtended region of a stenotic lesion. Proximally located lesions in the LAD have been associated with worse outcomes compared to distal LAD or non-LAD locations. ${ }^{31}$ A meta-analysis on 11,109 participants found that proximal lesions associated higher in-hospital and long-term adverse events compared to non-proximal plaques, after PCI. Also, mortality was higher in proximal LAD lesions compared to proximal RCA lesions that were percutaneously revascularized. ${ }^{32}$ Coronary dominance has also been shown to influence the severity of coronary lesions and patient outcomes following percutaneous revascularization procedures. A study that enrolled 
27,289 ACS patients reported that left coronary dominance was linked to an increased mortality rate over the course of 3.5 years after cardiac catheterization. ${ }^{33}$ These results were also validated by a larger population study on ACS subjects undergoing PCI, in which left dominance or co-dominance was associated with an higher in-hospital death rate. ${ }^{34}$

\section{CONCLUSIONS}

In conclusion, vulnerable plaques arising from the right coronary artery are longer, more voluminous and with larger lipid and non-calcified content, whereas those located in the left anterior descending artery present a higher volume of calcium, but also a higher degree of vulnerability. The higher vulnerability degree of vulnerable plaques located in the left anterior descending artery was illustrated by a larger number of CCTA-derived vulnerability markers with a specific distribution of calcium within the plaque depicted as spotty calcifications and napkinring phenotype. The least vulnerable lesions were present in the circumflex artery. Further research is required to assess the effect of hemodynamic characteristics according to plaque location, and also the role of site-specific phenotypes of vulnerable plaques on patient outcomes.

\section{CONFLICT OF INTEREST}

Nothing to declare.

\section{DISCLOSURE}

The results of the current research were presented during the EuroEcho Imaging Congress of the European Society of Cardiology in 2019, Vienna.

\section{ACKNOWLEDGEMENT}

This research has been funded by the research grant PlaqueImage, contract number 26/01.09.2016, SMIS code 103544, Project funded by the European Union and the Government of Romania through the Ministry of European Funds, and the Doctoral School of the "George Emil Palade" University of Medicine, Pharmacy, Science and Technology of Târgu Mureș, Romania.

\section{REFERENCES}

1. World Health Organization (WHO). Cardiovascular diseases (CVDs). Available at: https://www.who.int/news-room/factsheets/detail/cardiovascular-diseases-(cvds)
2. Libby P, Buring JE, Badimon L et al. Atherosclerosis. Nat Rev Dis Primers. 2019;5:56. doi: 10.1038/s41572-019-0106-z.

3. Shi X, Gao J, Lv Q et al. Calcification in Atherosclerotic Plaque Vulnerability: Friend or Foe? Front Physiol. 2020;11:56. doi: 10.3389/fphys.2020.00056.

4. Choi SY, Mintz GS. What have we learned about plaque rupture in acute coronary syndromes? Curr Cardiol Rep. 2010;12:338343. doi: 10.1007/s11886-010-0113-X.

5. Partida RA, Libby P, Crea F, Jang IK. Plaque erosion: a new in vivo diagnosis and a potential major shift in the management of patients with acute coronary syndromes. Eur Heart J. 2018;39:2070-2076. doi: 10.1093/eurheartj/ehx786.

6. Stefanadis C, Antoniou CK, Tsiachris D, Pietri P. Coronary Atherosclerotic Vulnerable Plaque: Current Perspectives. J Am Heart Assoc. 2017;6:e005543. doi: 10.1161/JAHA.117.005543.

7. Naghavi $M$, Libby $P$, Falk E, et al. From vulnerable plaque to vulnerable patient: a call for new definitions and risk assessment strategies: Part I. Circulation. 2003;108:16641672. doi: 10.1161/01.CIR.0000087480.94275.97.

8. Bentzon JF, Otsuka F, Virmani R, Falk E. Mechanisms of plaque formation and rupture. Circ Res. 2014;114:1852-1866. doi: 10.1161/CIRCRESAHA.114.302721.

9. Hafiane A. Vulnerable Plaque, Characteristics, Detection, and Potential Therapies. J Cardiovasc Dev Dis. 2019;6:26. doi: 10.3390/jcdd6030026.

10. Costopoulos C, Brown AJ, Teng Z, Hoole SP, West NE, Samady H, Bennett MR. Intravascular ultrasound and optical coherence tomography imaging of coronary atherosclerosis. Int J Cardiovasc Imaging. 2016;32:189-200. doi: 10.1007/ S10554-015-0701-3.

11. Rathod KS, Hamshere SM, Jones DA, Mathur A. Intravascular Ultrasound Versus Optical Coherence Tomography for Coronary Artery Imaging - Apples and Oranges?. Interv Cardiol. 2015;10:8-15. doi: 10.15420/icr.2015.10.1.8.

12. Knuuti J, Wijns W, Saraste A, et al. ESC Scientific Document Group. 2019 ESC Guidelines for the diagnosis and management of chronic coronary syndromes. Eur Heart J. 2020;41:407-477. doi: 10.1093/eurheartj/ehz425.

13. Benedek T, Jako B, Benedek I. Plaque quantification by coronary CT and intravascular ultrasound identifies a low CT density core as a marker of plaque instability in acute coronary syndromes. Int Heart J. 2014;55:22-28. doi: 10.1536/ihj.13-213.

14. Bittner DO, Mayrhofer T, Puchner SB, et al. Coronary Computed Tomography Angiography-Specific Definitions of HighRisk Plaque Features Improve Detection of Acute Coronary Syndrome. Circ Cardiovasc Imaging. 2018;11:e007657. doi: 10.1161/CIRCIMAGING.118.007657.

15. Murgia A, Balestrieri A, Crivelli P, et al. Cardiac computed tomography radiomics: an emerging tool for the non-invasive assessment of coronary atherosclerosis. Cardiovasc Diagn Ther. 2020;10:2005-2017. doi: 10.21037/cdt-20-156.

16. Liu W, Zhang Y, Yu CM, et al. Current understanding of coronary artery calcification. J Geriatr Cardiol. 2015;12:668675. doi: 10.11909/j.issn.1671-5411.2015.06.012.

17. van Rosendael AR, Narula J, Lin FY, et al. Association of HighDensity Calcified $1 \mathrm{~K}$ Plaque With Risk of Acute Coronary Syndrome. JAMA Cardiol. 2020;5:282-290. doi: 10.1001/ jamacardio.2019.5315.

18. Shmilovich H, Cheng VY, Tamarappoo BK, et al. Vulnerable plaque features on coronary CT angiography as markers of inducible regional myocardial hypoperfusion from severe 
coronary artery stenoses. Atherosclerosis. 2011;219:588-595. doi: 10.1016/j.atherosclerosis.2011.07.128.

19. Brutkiewicz A, Kruk M, Demkow M, et al. The natural history of napkin-ring sign by coronary computed tomography angiography. Postepy Kardiol Interwencyjnej. 2019;15:314320. doi: 10.5114/aic.2019.87886.

20. Opincariu D, Benedek T, Chițu M, Raț N, Benedek I. From CT to artificial intelligence for complex assessment of plaqueassociated risk. Int J Cardiovasc Imaging. 2020;36:2403-2427. doi: 10.1007/s10554-020-01926-1.

21. Katranas SA, Kelekis AL, Antoniadis AP, Ziakas AG, Giannoglou GD. Differences in Stress Forces and Geometry between Left and Right Coronary Artery: A Pathophysiological Aspect of Atherosclerosis Heterogeneity. Hellenic J Cardiol. 2015;56:217-223.

22. Giannoglou GD, Antoniadis AP, Chatzizisis YS, Louridas GE. Difference in the topography of atherosclerosis in the left versus right coronary artery in patients referred for coronary angiography. BMC Cardiovasc Disord. 2010;10:26. doi: 10.1186/1471-2261-10-26.

23. Asakura T, Karino T. Flow patterns and spatial distribution of atherosclerotic lesions in human coronary arteries. Circ Res. 1990;66:1045-1066. doi: 10.1161/01.res.66.4.1045.

24. Malek AM, Alper SL, Izumo S. Hemodynamic shear stress and its role in atherosclerosis. JAMA. 1999;282:2035-2042. doi: 10.1001/jama.282.21.2035.

25. Tuzcu EM, Kapadia SR, Tutar E, et al. High prevalence of coronary atherosclerosis in asymptomatic teenagers and young adults: evidence from intravascular ultrasound. Circulation. 2001;103:2705-2710. doi: 10.1161/01.cir.103.22.2705.

26. Gebhard C, Fuchs TA, Stehli J, et al. Coronary dominance and prognosis in patients undergoing coronary computed tomographic angiography: results from the CONFIRM (COronary CT Angiography EvaluatioN For Clinical Outcomes: An InteRnational Multicenter) registry. Eur Heart J Cardiovasc Imaging. 2015;16:853-862. doi: 10.1093/ehjci/jeu314.

27. Veltman CE, de Graaf FR, Schuijf JD, et al. Prognostic value of coronary vessel dominance in relation to significant coronary artery disease determined with non-invasive computed tomography coronary angiography. Eur Heart J. 2012;33:13671377. doi: 10.1093/eurheartj/ehs034.

28. Tuzcu EM, Kapadia SR, Tutar E, et al. High prevalence of coronary atherosclerosis in asymptomatic teenagers and young adults: evidence from intravascular ultrasound. Circulation. 2001;103:2705-2710. doi: 10.1161/01.cir.103.22.2705.

29. Motoyama S, Sarai M, Harigaya H, et al. Computed tomographic angiography characteristics of atherosclerotic plaques subsequently resulting in acute coronary syndrome. J Am Coll Cardiol. 2009;54:49-57. doi: 10.1016/j.jacc.2009.02.068.

30. Thomsen C, Abdulla J. Characteristics of high-risk coronary plaques identified by computed tomographic angiography and associated prognosis: a systematic review and metaanalysis. Eur Heart J Cardiovasc Imaging. 2016;17:120-129. doi: 10.1093/ehjci/jev325.

31. Elsman P, van 't Hof AW, Hoorntje JC, et al. Effect of coronary occlusion site on angiographic and clinical outcome in acute myocardial infarction patients treated with early coronary intervention. Am J Cardiol. 2006;97:1137-1141. doi: 10.1016/j. amjcard.2005.11.027.

32. Tang B, Yang H. Post percutaneous coronary interventional outcomes on proximal vs non-proximal lesions of the left and right coronary arteries: A systematic review and metaanalysis. Medicine (Baltimore). 2019;98:e16905. doi: 10.1097/ MD.0000000000016905.

33. Goldberg A, Southern DA, Galbraith PD, Traboulsi M, Knudtson ML, Ghali WA; Alberta Provincial Project for Outcome Assessment in Coronary Heart Disease (APPROACH) Investigators. Coronary dominance and prognosis of patients with acute coronary syndrome. Am Heart J. 2007;154:11161122. doi: 10.1016/j.ahj.2007.07.041.

34. Parikh NI, Honeycutt EF, Roe MT, et al. Left and codominant coronary artery circulations are associated with higher inhospital mortality among patients undergoing percutaneous coronary intervention for acute coronary syndromes: report From the National Cardiovascular Database Cath Percutaneous Coronary Intervention (CathPCI) Registry. Circ Cardiovasc Qual Outcomes. 2012;5:775-782. doi: 10.1161/ CIRCOUTCOMES.111.964593. 